\title{
Analysis and Intervention of Severe Acute Malnutrition in Children at an Urban Public School in Mumbai, India
}

\author{
Esha Chainani \\ Department of Obstetrics and Gynaecology, Dr. D Y Patil Hospital and Research Centre, Navi Mumbai, India \\ Email: eshachainani@hotmail.com
}

How to cite this paper: Chainani, E. (2018) Analysis and Intervention of Severe Acute Malnutrition in Children at an Urban Public School in Mumbai, India. Open Journal of Preventive Medicine, 8, 131-141. https://doi.org/10.4236/ojpm.2018.84013

Received: February 8, 2018

Accepted: April 25, 2018

Published: April 28, 2018

Copyright $\odot 2018$ by author and Scientific Research Publishing Inc. This work is licensed under the Creative Commons Attribution International License (CC BY 4.0).

http://creativecommons.org/licenses/by/4.0/

\section{c) (i) Open Access}

\begin{abstract}
Background: There is a dearth of information regarding trends of malnutrition and weight status in children from rapidly developing economies like India. Objectives. The aim of this study is to analyze the dynamics of malnutrition in a group of school children from India, and then provide interventions for the children that have severe acute malnutrition. Methods. Children that were found to lie between -3 to -4 SD of parameters of BMI, height for age and weight for age were labeled as SAM. Children that were screened for wasting and fell between -2 to $-4 \mathrm{SD}$ were also included under the label of SAM. All participants were also given a prototype 1500 -calorie diet to follow for the entire duration and 6 weekly sessions were taken for the parents on various topics on nutrition. Results. Quantitative analysis showed a great improvement in weights and heights of severely malnourished children. The average increase in weight of all children that participated in the study was $9.09 \%$ and the average increase in height was $1.39 \%$. The weekly sessions given to the parents on various nutrition topics could be the cause of high compliance to the diet given to the children. Conclusion: Malnutrition is still a very big problem in developing countries like India. Basic interventions like an improvement in diet, education about micronutrients and balanced diet, and food-based interventions in severe malnutrition can lead to a great improvement in the health and development status of children.
\end{abstract}

\section{Keywords}

Malnutrition, Children, Sam, India, Nutrition

\section{Introduction}

There is a dearth of information regarding trends of malnutrition and weight 
status in children from rapidly developing economies like India. Severe and widespread undernutrition in low-income countries like India continues to exert enormous cost in terms of survival among infants and young children, though the actual figures of severely malnourished children are not well known even in metropolitan cities like Mumbai. Governments in developing countries have invested significant resources in various policies and social programs to tackle this problem. However, there is a continuing need to ensure a more efficient use of these resources. Interventions include the provision of nutritional supplements, subsidized prices, cash benefits, and childcare programs. Despite these efforts, child care remains a public health priority [1].

The aim of this study is to analyze the dynamics of malnutrition in a group of school children from India, and then provide interventions for the children that have severe acute malnutrition. For children under 60 months, WHO and UNICEF recommend the use of a cut-off for weight-for-height of below -3 standard deviations (SD) of the WHO standards to identify infants and children as having SAM [2]. In children above the age of 60 months, WHO growth standards [3] were used. Values below -3 SD identify children of severe acute malnutrition.

This study endeavors to identify the state of undernutrition in children between the ages of 4 - 8 years in Shindewadi Mumbai Public School, by using the WHO growth standards and subsequently improve their collective nutrition levels, knowledge about health and dietary intake. Severely malnourished children that would be identified in this study would undergo implementation of food based interventions for the duration of this project.

Food-based interventions have been shown to have several benefits including providing additional energy, protein, and nutrients in a familiar form that can be considered in the usual diet [4].

Dietary behaviors of students need to be corrected in order to reach better performance and health. The improvement of knowledge of health and diet is as significant as dietary intake has an important role in improving physical and mental function, as well as the learning abilities of children. Malnutrition might also be the cause of several problems in the trend of growth and can accelerate cognitive impairment [5]. Therefore considering the nutritional status of school-aged children could be important in this regard.

\section{Methodology}

\section{Subjects}

The Nutrition Project took place at Shindewadi Mumbai Public School in a municipal school in Mumbai for low income families between $29^{\text {th }}$ January 2017 and $11^{\text {th }}$ March 2017. This was a 6 week project to screen for severe malnutrition and provide interventions to improve the nutrition status of these children whilst educating the parents about nutrition and balanced diet.

Inclusion criteria for the study were all students of the Shindewadi Mumbai 
Public School that had no allergies, no prolonged absenteeism from school, no congenital or chronic illnesses, absence of any infection or fever, and any vomiting or diarrhea during the course of the interventions.

There were 144 children in the Shindewadi Mumbai Public School from Jr. Kg to $2^{\text {nd }}$ grade out of which 2 students that were allergic to peanuts, 3 students were absent for more than 15 days and could not be monitored, and 4 students complained of chronic illnesses (lasting more than 3 weeks) and hence were excluded from the study.

135 children between the ages of $4-8$ years in Jr. $\mathrm{Kg}$ to $2^{\text {nd }}$ grade as seen in Figure 1 were analyzed as part of the study by simple cluster sampling. There were a total of 62 girls and 73 boys as seen in Figure 2 .

\section{Design}

The parameters used in this study to screen for malnutrition were WHO growth standards of: BMI (body mass index) for age, height for age, weight for age and, wasting (weight for height, only for children under 60 months of age). Children that were found to lie between -3 to -4 SD of BMI for age, height for

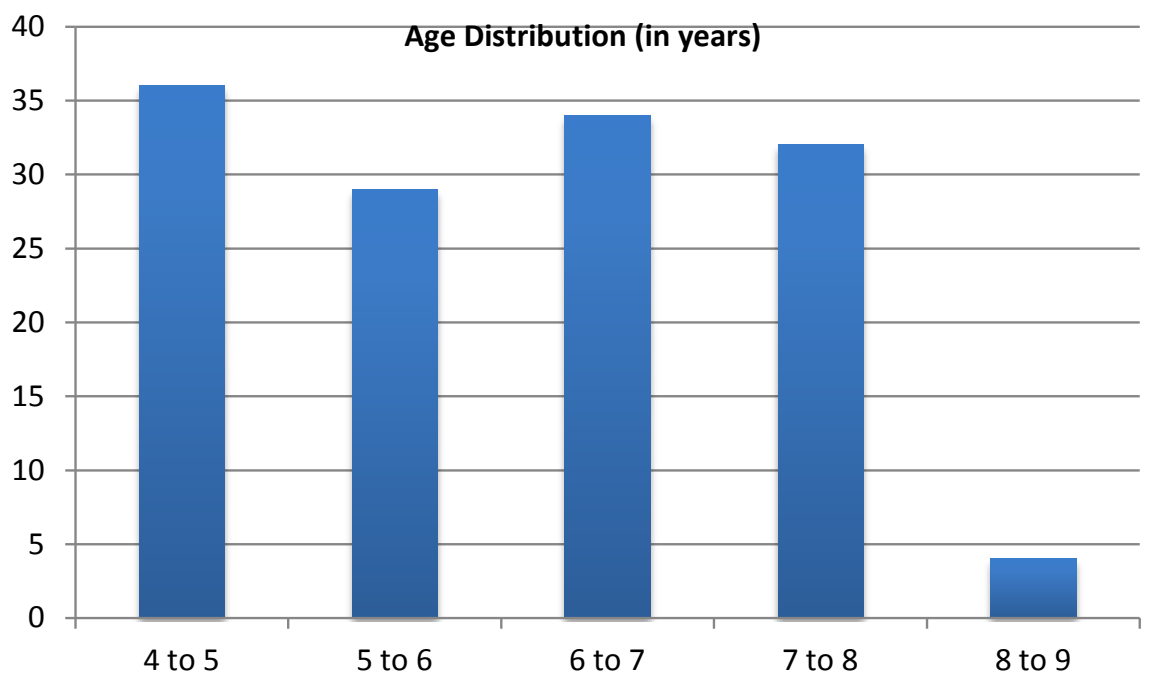

Figure 1. Age distribution of participants undergoing the study.

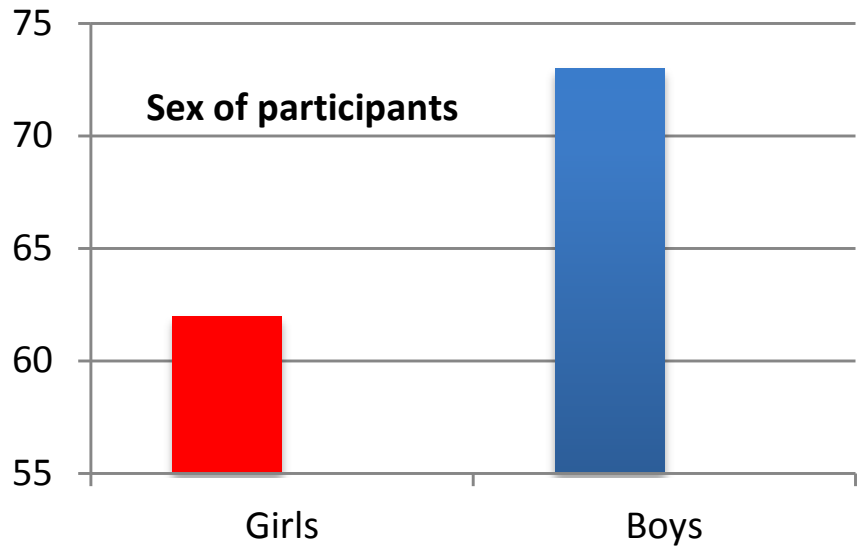

Figure 2. Sex of participants undergoing the study. 
age and weight for age were labeled as SAM. Children under the age of 60 months that were screened for wasting (weight for height) and fell between -2 to -4 SD were also included under the label of SAM.

MUAC (middle upper arm circumference) is age dependent in infants and children over the age of 4 and hence was not used as most of the students were older than 4 years. Head circumference, triceps skin fold for age and chest circumference is used only up to 60-month-old children as per the WHO standards and was not used in this study.

To measure these parameters height and weight of the children were taken on the $29^{\text {th }}$ January and $11^{\text {th }}$ march 2016 . These data were collected under research conditions by a single observer to minimize measurement errors. Data was double entered and verified manually. It was analyzed by the WHO growth standards. Any information on illnesses and allergies were recorded. Informed consent was taken from all the parents of the children in the study.

\section{Anthropometrics}

At the start of the intervention (base-line survey), and after 6 weeks of intervention, height and weight of all children was determined. This height and weight was used for the WHO growth standards [3] of weight for height, BMI for age, weight for age, and height for age.

Control

A diet recall sheet was given to parents before the start of the project to get an idea of what the child's diet resembled. For the project, the parents were given a prototype diet of 1500 calories per day as a definitive meal plan to be followed for the child. All of the food would be made at home, no packaged food or drink was to be taken by the child for the duration of the project. This was prescribed to all children as a control plan for the 20 SAM children who would be given an intervention model.

The World Health Organization (WHO) recommends treating all school children at regular intervals with deworming drugs in areas where helminth infection is common as they state this will improve health, intellect, and school attendance while decreasing child mortality [6]. Thus, for all children 2 days before the start of the project a deworming tablet was given (Albendazole $400 \mathrm{mg}$ ) as a single chewable oral tablet.

For all children, a multi-vitamin syrup was given to take $5 \mathrm{ml}$ once a day every day for the entire duration of the 6 week project.

For all the parents of the children in the project, 6 one hour weekly sessions were taken. In these sessions apart from the topic covered, any questions or doubts by the parents were explained to them and all follow up questions and concerns were addressed.

Session 0 (before the start of the project)-Explanation of the project and signing of informed consent.

Session 1-malnutrition: causes, symptoms, effects and treatment.

Session 2-importance and components of balanced diet. 
Session 3-micronutrients: importance and micronutrient deficiency diseases.

Session 4-personal hygiene and sanitation.

Session 5-food safety.

Session 6-importance of continuing these diet changes, results of the project and any changes seen.

\section{Intervention}

An intervention schedule was followed for the children that were SAM ( -3 to $-4 \mathrm{SD}$ of $\mathrm{BMI}$, weight for age, height for age) and ( -2 to $-4 \mathrm{SD}$ of wasting criteria).

There were a total of 20 children that fell under these criteria. Along with the control protocol, they were additionally given an intervention of Additional Supplementary foods ("Laddoos"), the ingredients of which were paid for by NGO KaRo. These Additional Supplementary foods ("Laddoos") were ready to eat, made with the participation of the parents and school administration. Ingredients of the Laddoos were $30 \mathrm{~g}$ milk powder, $20 \mathrm{~g}$ roasted groundnuts, $28 \mathrm{~g}$ of sugar, $20 \mathrm{~g}$ of oil bringing it to a total of $98 \mathrm{~g}$ per day. They were made by the school administration every day and given in two divided Laddoos twice a day at 9 am and 2 pm under the supervision of the school coordinator, principal and teachers. On days the school was off these Laddoos would be made and sent with the parents who would then supervise their consumption at the same timings of 9 am and $2 \mathrm{pm}$.

Notations were made if there was any adverse reaction, allergy or illness during the 6 week period.

\section{Analysis and statistics}

Data presented as mean \pm SD. Input data was calculated based on WHO growth standards of BMI for age, height for age, weight for age and wasting. This data was then divided into zones of standard deviations of -3 to $-4,-2$ to -3 , and so on. This was then sub-divided into girls and boys. Input data for the start date and end date was then compared and contrasted to find out changes in growth standards of the children.

\section{Results}

A total of 135 children were a part of the study, with $45.5 \%$ being girls and $54.5 \%$ as boys with the mean age of 6 years (ages between $4-8$ years). Their height and weight was measured on day 1 of the program and day 40 of the program.

36 children were below the age of 5 years ( 60 months) and hence were eligible to be measured for weight for height (wasting).

135 children were measured for WHO growth standards of weight for age, BMI for age and height for age.

\section{BMI for age}

7 children ( 3 girls and 4 boys) were in the severely malnourished group of -3 to -4 SD on day 1.2 children ( 0 girl and 2 boys) were in the severely malnourished group of -3 to -4 SD on day 40 . 
21 children ( 8 girls and 13 boys) were at or above the mean on day 1 and 29 children ( 9 girls and 20 boys) were at or above the mean on day 40 as seen in Figure 3.

\section{Weight for age}

12 children ( 3 girls and 9 boys) were in the severely malnourished group of -3 to $-4 \mathrm{SD}$ on day 1.3 children ( 0 girl and 3 boys) were in the severely malnourished group of -3 to -4 SD on day 40 .

7 children ( 2 girls and 5 boys) were at or above the mean on day 1 and 8 children ( 3 girls and 5 boys) were at or above the mean on day 40 as seen in Figure 4.

\section{Height for age}

4 children ( 1 girl and 3 boys) were in the severely malnourished group of -3 to $-4 \mathrm{SD}$ on day 1.3 children ( 0 girl and 3 boys) were in the severely malnourished group of -3 to -4 SD on day 40 .

3 children ( 0 girl and 3 boys) were at or above the mean on day 1 and 9 children ( 3 girls and 6 boys) were at or above the mean on day 40 as seen in Figure 5.

\section{Weight for height ( Wasting)}

36 children (14 girls and 22 boys) were below the age of 60 months.

7 children ( 4 girls and 3 boys) were in the severely malnourished group of -2 to $-4 \mathrm{SD}$ on day 1.0 children ( 0 girl and 0 boys) were in the severely malnourished group of -2 to -4 SD on day 40 .

2 children ( 0 girl and 2 boys) were at or above the mean on day 1 and 7 children ( 1 girl and 6 boys) were at or above the mean on day 40 as seen in Figure 6.

In total, the average increase in weight of all children that participated in the study was $9.09 \%$ ( $4.6 \%$ in girls and $12 \%$ in boys). The average increase in height was $1.39 \%$ ( $1.05 \%$ in girls and $1.07 \%$ in boys).

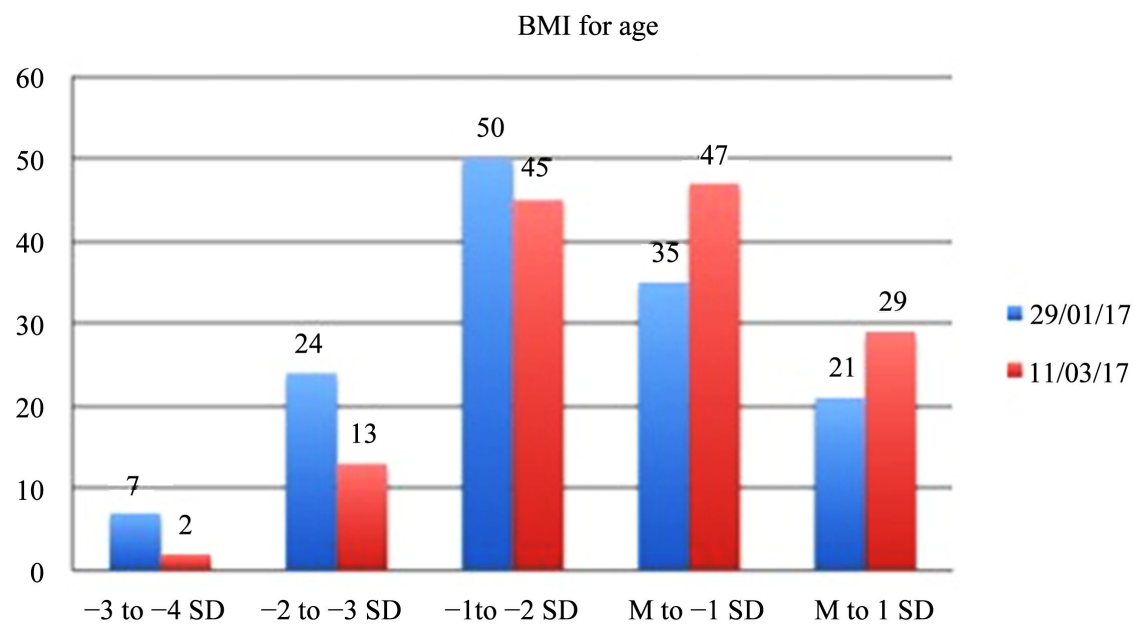

Figure 3. Comparison of BMI for age in study participants at day 1 of the study with data on day 42 of the study. 
Weight for age

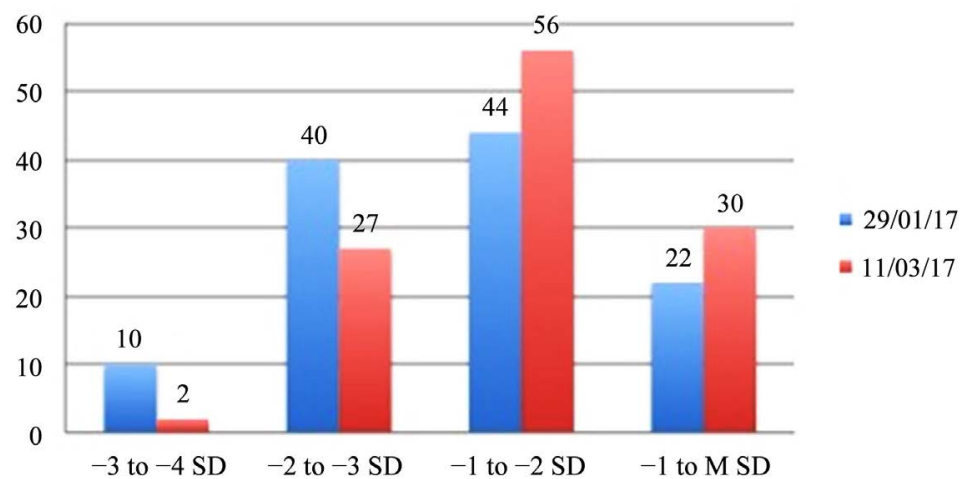

Figure 4. Comparison of weight for age in study participants at day 1 of the study with data on day 42 of the study.

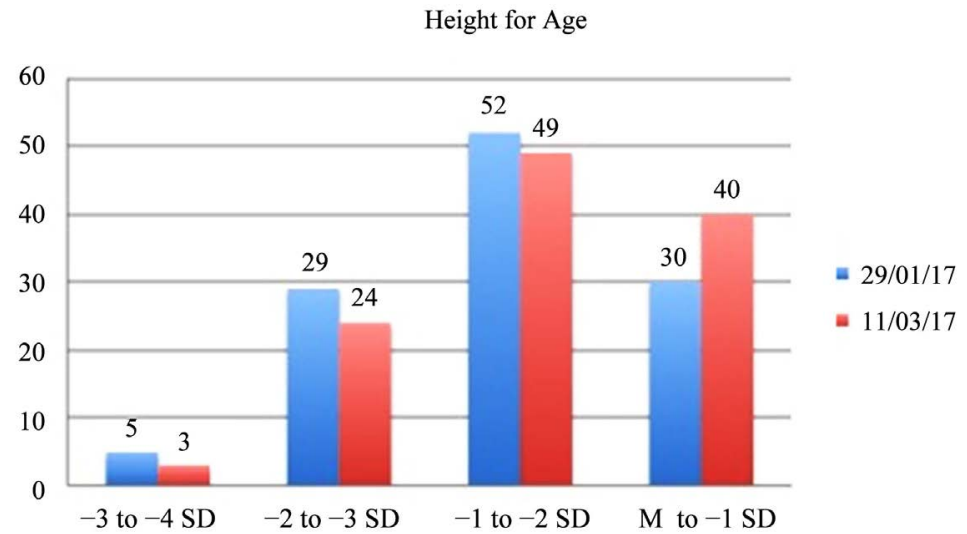

Figure 5. Comparison of height for age in study participants at day 1 of the study with data on day 42 of the study.

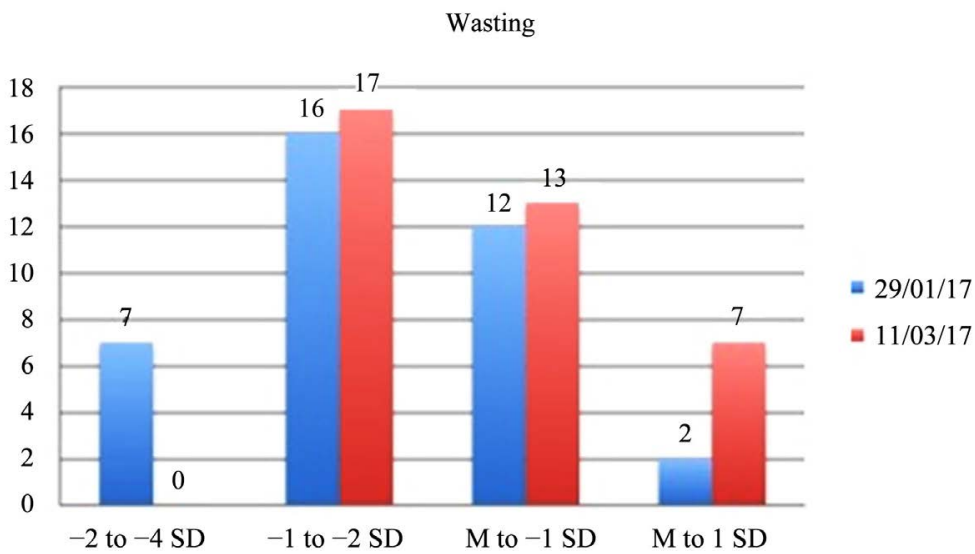

Figure 6. Comparison of wasting (weight for height) in study participants younger than 60 months of age at day 1 of the study with data on day 42 of the study.

Data taken from the parents of the students in the study in form of a questionnaire showed 56.2\% of the population living in "kaccha" (crude/temporary/semi-permanent) houses as seen in Figure 7, poor sanitation facilities (open defecation) in $24.5 \%$ of 
the population, with only $11.8 \%$ reporting a toilet in their home as seen in Figure $8.76 .2 \%$ of the population consumed tap water without any filtering or boiling as seen in Figure 9.

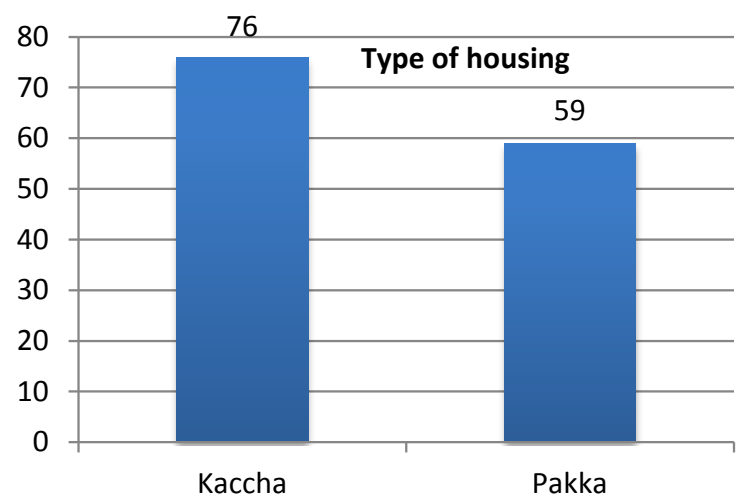

Figure 7. Population of study participants residing in types of houses.

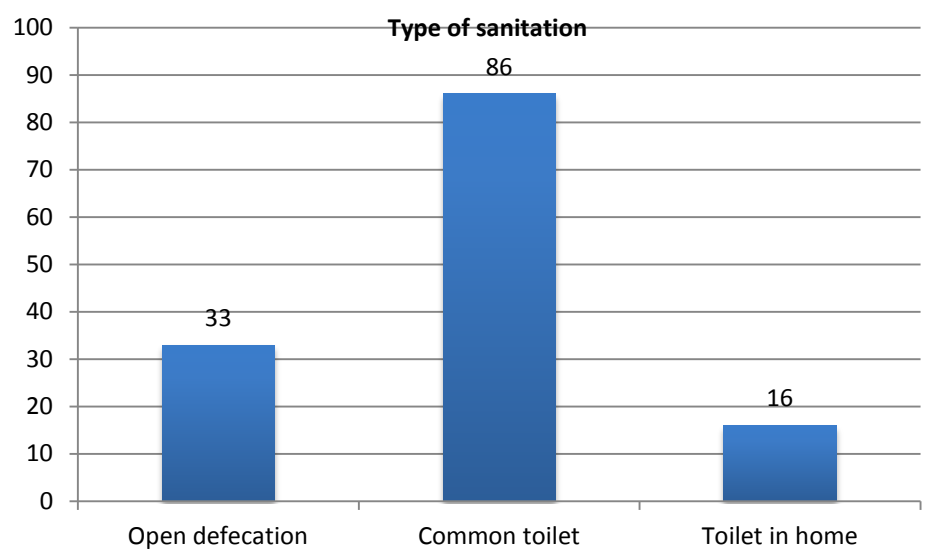

Figure 8. Population of study participants with different types of sanitation methods in their homes.

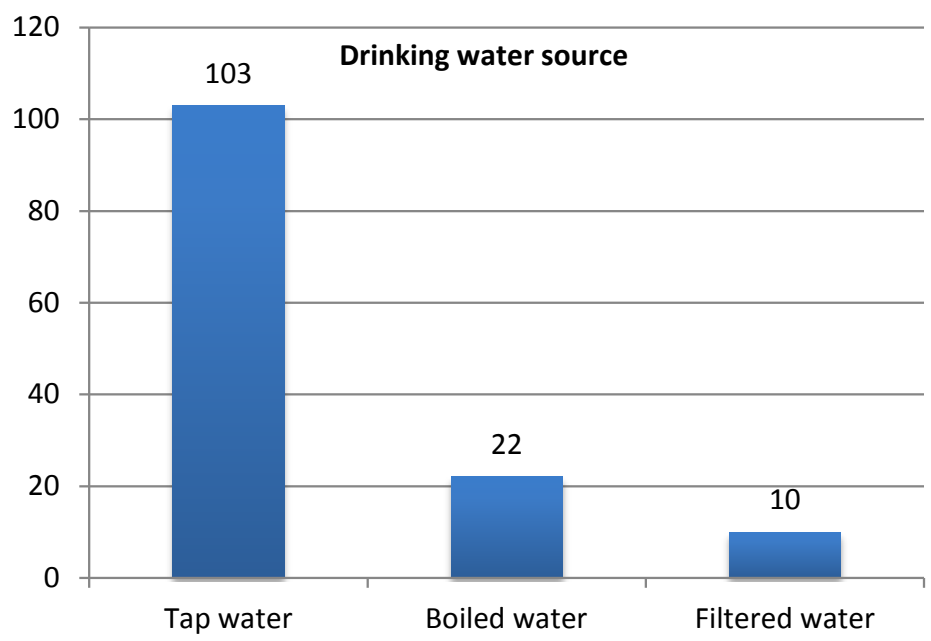

Figure 9. Population of study participants with different drinking water sources. 


\section{Discussion}

This study has shown an improvement in the nutritional status of children in as short a timeframe as 6 weeks, the possibilities for their improvement over a longer time period if this project can be sustained are promising. Dietary intake has an important role in improving physical and mental function as well as the learning abilities of children [7]. Therefore considering the nutritional intakes of school-aged children could be important in this regard [8].

Quantitative analysis showed a great improvement in weights and heights of the severely malnourished children. It also showed an average increase in weight and height of all the children that were in the study and were just subjected to a change in diet and introduction of multivitamin syrup. The weekly informative sessions given to the parents to educate them on various nutrition topics could be the cause of high compliance to the diet given to the children.

Teachers also qualitatively reported the benefits on the physical function and school performance of the children. Increased concentration in school, better scores on class tests and lower absentee rates were also reported.

Underweight children have been seen to come from families that practice poor hygiene-notably in this case a lack of sanitation and accessibility to safe water. Most of the parents were not aware of the dangers of drinking tap water without any filtration process. Proper hand washing before consuming any food was not understood by parents and hence was not explained to the students. These families live in urban slums in India and did not understand the gravity of the situation when it came to their children being underweight and malnourished. All of the parents reported giving children sweets, chips and other junk foods as they did not understand the importance of nutrients and a balanced diet. However this was explained in the 6 sessions that were held for the parents. Once they were explained nutrients, balanced diet, malnutrition and associated disorders, food safety and hygiene they were very careful with the food given to the children and adopted more sanitary and hygienic practices. Many studies have reported that poor sanitation status, lack of personal hygiene and low socioeconomic statuses have an impact on malnutrition [9].

Multivitamin syrups and the ingredients of the intervention food given to the students are also cost effective and can be scaled at a larger level. The ingredients for the intervention food are easily available in Mumbai and their cost can be managed by even low income families. Thus making the process of treatment of severe acute malnutrition possible and accessible in all sections of society in developing countries like India. This analysis and intervention model is scalable and useful for all other developing countries as well and studies like this can be used to affect change at global grassroots level.

Malnutrition and susceptibility to infections are closely related and could result in a vicious cycle. Among the children in this study malnutrition was abundant and also the reported health related problems were high. Incidence of parents diagnosed colds, sore throats and diarrhea decreased during the study in all children. Enhanced supply of macro- and micronutrients clearly showed its ben- 
efits in countering the vicious cycle of malnutrition and illness [10].

This study stresses on the fact that a little education can go a long way. Parents in low socioeconomic strata of society in developing countries like India do not have the knowledge of basic nutrition and hygiene. Communication about the above can change the way entire families behave toward malnutrition and hence towards health. Understanding how better sanitation and hygiene along with a balanced diet can change their and their children's lives is a real impetus for them to act on it. Education and proper training for breastfeeding and weaning to mothers would also be a step in combating malnutrition. There are modifiable and non-modifiable factors to malnutrition, out of which the environmental factor is one that can be worked on in a cost effective way. The quality of the future of human resources depends on the children of today [9] we must do everything we can to ensure their optimum health.

As a limitation in this study blood samples of the students could not be collected. It is suggested to also collect blood before and after these supplementary feeding programs to definitively measure the micronutrient deficiencies in future studies for analyzing the nutritional status. Collaborative activities of the school administration were the most important strength of this program.

\section{Conclusion}

The pediatric population is experiencing a rapid growth and nutritional transition. Malnutrition is still a very big problem in developing countries like India, due to food scarcity and low education of nutrition and dietary guidelines in the lower socioeconomic sections of society. Basic interventions like an improvement in diet, education about micronutrients and balanced diet, and food based interventions in severe malnutrition can lead to a great improvement in the health and development status of the pediatric population in India. Feasible strategies are needed to quickly address the dietary inadequacies and malnutrition of children in India.

\section{Conflict of Interest}

None declared. Neither the funders nor school administrators had any role in the design, analysis or reporting of the results from this study.

\section{Acknowledgements}

We are grateful to the study participants and their families, The Akanksha Foundation, the teachers and administrators of Shindewadi Mumbai Public School for their support and implementation of this program in their school.

We would like to thank the NGO, KaRo for providing funding for the supplementary nutrition given to the severely malnourished children and their interest in this cause.

\section{References}

[1] Padilla, A. and Trujillo, J. (2015) An Impact Assessment of the Child Growth, De- 
velopment and Care Program in the Caribbean Region of Colombia. Cadernos de Saúde Pública, 31, 2099-2109. https://doi.org/10.1590/0102-311X00153514

[2] La Course, S., Chester, F., Preidis, G., et al. (2014) Lay-Screeners and Use of WHO Growth Standards Increase Case Finding of Hospitalized Malawian Children with Severe Acute Malnutrition. Journal of Tropical Pediatrics, 61, 44-53. https://doi.org/10.1093/tropej/fmu065

[3] WHO (2009) WHO Child Growth Standards. Developmental Medicine \& Child Neurology, 51, 1002-1002. https://doi.org/10.1111/j.1469-8749.2009.03503.x

[4] Djazayery, A., Rahmani, K., Pourshariari, M., Habibi, M. and Heydari, H. (2011) The Effects of a Daily Milk Supplement on the Mental Growth and School Performance of Female Primary School Children. Proceedings of the Nutrition Society, 70, E230. https://doi.org/10.1017/S0029665111002813

[5] Khadijeh Rahmani, L. (2011) Effects of Daily Milk Supplementation on Improving the Physical and Mental Function as Well as School Performance among Children: Results from a School Feeding Program. Journal of Research in Medical Sciences. The Official Journal of Isfahan University of Medical Sciences, 16, 469.

[6] Taylor-Robinson, D.C. (2016) Deworming Drugs for Soil-Transmitted Intestinal Worms in Children: Effects on Nutritional Indicators, Haemoglobin and School Performance.

http://cochranelibrary-wiley.com/doi/10.1002/14651858.CD000371.pub6/abstract

[7] Martorell, R. and Nguyen, P. (2010) Interrelationship between Growth and Development in Low and Middle Income Countries. Nestle Nutr Workshop Ser Pediatr Program, 65, 99-118. https://doi.org/10.1159/000281151

[8] Chen, L., Wahlqvist, M.L., Teng, N.C. and Lu, H.M. (2009) Imputed Food Insecurity as a Predictor of Disease and Mental Health in Taiwanese Elementary School Children. Asia Pacific Journal Of Clinical Nutrition, 18, 605-619

[9] Ansuya, Baby, S. and Nayak, B. (2018) Risk Factors for Malnutrition among Preschool Children in Rural Karnataka: A Case-Control Study. BMC Public Health, 18, 283. https://doi.org/10.1186/s12889-018-5124-3

[10] Lien do, T.K. (2009) Impact of Milk Consumption on Performance and Health of Primary School Children in Rural Vietnam. Asia Pacific Journal Of Clinical Nutrition, 18, 326-334. http://www.ncbi.nlm.nih.gov/pubmed/19786380 